University of Wollongong

Research Online

Faculty of Engineering and Information

Faculty of Engineering and Information

Sciences - Papers: Part A

Sciences

$1-1-2012$

\title{
An approach to compensate the object movement errors in phase shifting profilometry
}

Lei Lu

University of Wollongong, I1490@uowmail.edu.au

Jiangtao Xi

University of Wollongong, jiangtao@uow.edu.au

Yanguang Yu

University of Wollongong, yanguang@uow.edu.au

Limei Song

Tianjin Polytechnic University

Follow this and additional works at: https://ro.uow.edu.au/eispapers

Part of the Engineering Commons, and the Science and Technology Studies Commons

Research Online is the open access institutional repository for the University of Wollongong. For further information contact the UOW Library: research-pubs@uow.edu.au 


\title{
An approach to compensate the object movement errors in phase shifting profilometry
}

\author{
Abstract \\ Phase shifting profilometry (PSP) technique is widely used as a 3-D shape measurement technique due to \\ its robustness and accuracy. However, PSP requires multiple fringe pattern images to be projected onto \\ an object and a reference plane to calculate the phase value, and also the object must maintain \\ motionless when the measurement is taken. If the object moves during the measurement, significant \\ errors will be introduced when calculating the phase value. This paper analyses the relationship between \\ the object movement and the phase value, and proposes a method for compensating the errors caused \\ by two-dimensional movement of object. This method can eliminate the errors caused by two- \\ dimensional movement of object and reconstruct the object shape successfully. The effectiveness of the \\ proposed method is verified by simulations. (c) 2012 SPIE.
}

\section{Keywords}

shifting, profilometry, approach, compensate, object, movement, errors, phase

\section{Disciplines}

Engineering | Science and Technology Studies

\section{Publication Details}

L. Lu, J. Xi, Y. Yu \& L. Song, "An approach to compensate the object movement errors in phase shifting profilometry," in Proceedings of SPIE - The International Society for Optical Engineering 8563, Optical Metrology and Inspection for Industrial Applications II, 2012, pp. 856304-1-856304-8. 


\title{
An approach to compensate the object movement errors in phase shifting profilometry
}

\author{
Lei $\mathrm{Lu}^{\mathrm{a}}$, Jiangtao Xi*a, Yanguang $\mathrm{Yu}^{\mathrm{a}}$, Limei Song ${ }^{\mathrm{b}}$ \\ ${ }^{a}$ School of Electrical, Computer and Telecommunications Engineering, \\ University of Wollongong, Northfields Ave, Wollongong, NSW, 2522; ${ }^{\mathrm{b}}$ Computer Technic and \\ Autonatization College, Tianjin Polytechnic University, 63 Chenglin Road, Tianjin, China, 300160
}

\begin{abstract}
Phase shifting profilometry (PSP) technique is widely used as a 3-D shape measurement technique due to its robustness and accuracy. However, PSP requires multiple fringe pattern images to be projected onto an object and a reference plane to calculate the phase value, and also the object must maintain motionless when the measurement is taken. If the object moves during the measurement, significant errors will be introduced when calculating the phase value. This paper analyses the relationship between the object movement and the phase value, and proposes a method for compensating the errors caused by two-dimensional movement of object. This method can eliminate the errors caused by two-dimensional movement of object and reconstruct the object shape successfully. The effectiveness of the proposed method is verified by simulations.
\end{abstract}

Keywords: PSP, compensation, movement error, motion

\section{INTRODUCTION}

In recent years, fringe pattern profilometry (FPP) has been one of the most popular non-contact methods for 3-D shape measurement. In FPP, the fringe analysis method includes Fourier transform profilometry (FTP) ${ }^{[1]}$, phase shifting profilometry (PSP) ${ }^{[2]}$, modulation measurement profilometry(MMP $)^{[3]}$ and etc. FTP and PSP are two most typical algorithms among them. FTP requires only one or two fringe pattern images to calculate the phase value ${ }^{[4]}$, hence it is suitable for dynamic object measurement. However, FTP is sensitive to ambient light and reflectivity variations, so the accuracy will be easily affected. In contrast, PSP requires multiple fringe pattern images (normally at least three) ${ }^{[5]}$, but has the following advantages ${ }^{[6]}$ : (1) has a pixel-level measurement resolution due to point-by-point measurement; (2) less sensitive to surface reflectivity variations, can be used for complex surface object measurement; (3) less sensitive to ambient light. The proposed method in this paper is based on conventional PSP method.

PSP is a method that based on calculating the triangle function relationship among a sequence of phase shift fringe pattern images. According to the phase shift times N, PSP can be classified as N-step PSP (N $\geq 3$ ), such as three-step PSP or four-step PSP, the phase shift is $2 \pi / N$ for each step. The more steps are used, the less sensitive to the device nonlinear distortion ${ }^{[7]}$. For high-speed and accurate measurement of complex geometric shapes, three-step PSP is the first choice ${ }^{[5]}$.

However, there are still some problems to be addressed in PSP. PSP uses the intensity values of the phase shift fringe patterns to calculate the phase value, and the intensity values should have the same phase shift of $2 \pi / N$ for the same point on the object. It is always assumed that the object is motionless when the fringe patterns are being projected and captured. However, this assumption is impractical. Errors will be introduced if the object moves when the measurement is performing ${ }^{[5]}$. In practice, it is hard to guarantee the object is motionless even the period between the projection and the capture of fringe patterns is very short. Therefore, it is important to analyse and compensate the errors caused by object movement to achieve high accuracy.

*jiangtao@uow.edu.au

Optical Metrology and Inspection for Industrial Applications II, edited by Kevin G. Harding,

Peisen S. Huang, Toru Yoshizawa, Proc. of SPIE Vol. 8563, 856304 · (c) 2012 SPIE

CCC code: $0277-786 / 12 / \$ 18 \cdot$ doi: $10.1117 / 12.1000086$ 
There are some existing works on this field of research. Xianyu Su et al. used FTP to measure dynamic object ${ }^{[4]}$. They proposed an algorithm which defines the phase difference between two deformed fringe patterns, and only requires less than two fringe pattern images to calculate phase values, however it suffers from the noise caused by ambient light and reflectivity variation.

In 2007, Zhang and Yau proposed a modified two-plus-one phase shift algorithm to reduce the errors due to motion ${ }^{[5]}$, which is based on the method proposed by Angel and Wizinowich. Zhang and Yau used two fringe pattern images that have $90^{\circ}$ phase shift and a uniform flat image as the third image to calculate the phase value. Because the phase information is contained in all $\mathrm{N}$ fringe pattern images in N-step PSP, and only in two fringe pattern images in two-plusone method, the errors due to motion are less. However, errors still can be introduced when the object moves in the period of the two fringe pattern images projecting.

Wang and Zhang et al. combined the conventional PSP with the gray code to solve errors caused by rapidly moving object $^{[8]}$. They can measure multiple rapidly moving objects simultaneously but still cannot reduce the inherent phase error caused by movement.

This paper analyses the relationship between object movement and its corresponding change of phase value. Normally two-dimensional movement can be divided into horizontal movement and vertical movement, hence the relationship between phase value and horizontal movement or vertical movement will be analysed firstly. Based on the relationship, we propose a compensation method to address the errors caused by two-dimensional movement of object. The compensation method can eliminate the errors caused by any two-dimensional movement, the shape of the object can be successfully reconstructed, and the high accuracy measurement for moving object can be achieved.

This paper is organized as follows. Section 2 describes the principle of PSP and the compensation method. In section 3, simulations are given to verify the proposed method. Section 4 concludes this paper.

\section{PRINCIPLE}

In PSP, a series of phase shifted sinusoidal fringe patterns are projected to the object and the reference plane by the projector and then captured by camera. Calculating the triangulation function on the intensity values of fringe patterns, we can obtain the phase values for each $\operatorname{pixel}^{[9]}$. The obtained phase values are limited from $-\pi$ to $\pi$, so, they are called wrapped phase and $2 \pi$ discontinuities can be observed. A phase unwrapping algorithm is used to remove the discontinuities and retrieve continuous phase values which called unwrapped phase ${ }^{[10]}$. At last, the 3-D information can be calculated by the unwrapped phase difference between object and reference plane.

\subsection{N-step PSP algorithm}

The fringe patterns of N-step PSP algorithm can be expressed as

$$
I_{n}(x, y)=I^{\prime}(x, y)+r(x, y) \cos \left[\phi(x, y)+\frac{2 \pi(n-1)}{N}\right] \quad n=1,2, \ldots, N
$$

Where $\mathrm{n}$ is the index of step number; $I_{n}(x, y)$ is the intensity value of $\mathrm{n}$-th fringe pattern image; $I^{\prime}(x, y)$ represents the average intensity; $r(x, y)$ is the intensity modulation; $\phi(x, y)$ is the wrapped phase value needs to be solved. It is obvious that the intensity values between different steps have the same phase shift $2 \pi / N$.

A general formula for calculating the wrapped phase is ${ }^{[2]}$

$$
\phi(x, y)=\tan ^{-1} \frac{I_{A}}{I_{B}}
$$

Where $I_{A}$ and $I_{B}$ is 


$$
\begin{gathered}
I_{A}=-\sum_{n=1}^{N} I_{n}(x, y) \sin \frac{2 \pi(n-1)}{N} \\
I_{B}=\sum_{n=1}^{N} I_{n}(x, y) \cos \frac{2 \pi(n-1)}{N}
\end{gathered}
$$

From equation 2, 3 and 4 we can know that the wrapped phase is calculated from the intensity values of different fringe pattern images. The wrapped phase $\phi(x, y)$ is ranging from $-\pi$ to $\pi$, after unwrapping, the unwrapped phase $\Phi(x, y)$ can be expressed by

$$
\Phi(x, y)=2 \pi k(x, y)+\phi(x, y)
$$

The unwrapping algorithm ${ }^{[10]}$ is aimed to find integer number $k(x, y)$, which means the period order of the fringes. At last, by using the unwrapped phase difference between the object and the reference plane, the height information of the object can be calculated using equation 6

$$
h(x, y)=\frac{l_{0} \Phi^{d}(x, y)}{\Phi^{d}(x, y)-2 \pi f_{0} d_{0}} \quad \Phi^{d}(x, y)=\Phi^{o}(x, y)-\Phi^{r}(x, y)
$$

$\Phi^{o}(x, y)$ and $\Phi^{r}(x, y)$ is the unwrapped phase of the object and the reference plane respectively, $\Phi^{d}(x, y)$ is the difference of them. $l_{0}$ is the distance between camera and reference plane, $d_{0}$ is the distance between camera and projector, $f_{0}$ is the spatial frequency of the fringe pattern.

\subsection{Principle of compensation method for two-dimensional movement}

Equation 2 only can be used when the object is motionless during the measurement. Errors will be introduced if the object moves during the measurement. In order to address this problem, the relationship between two-dimensional movement and phase value needs to be analysed firstly.

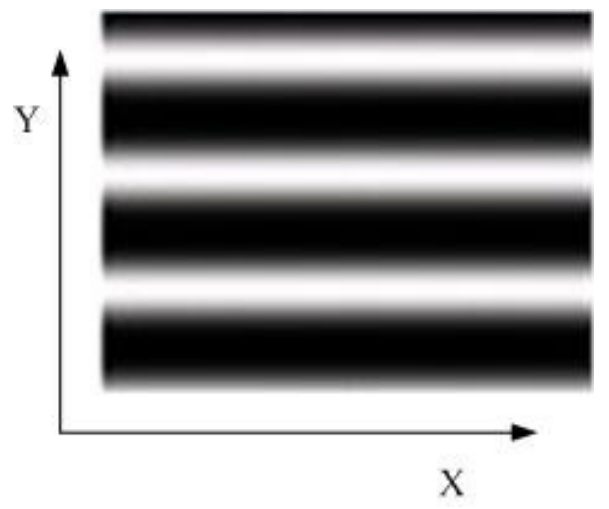

Figure 1. Horizontal fringe pattern.

Figure1 shows the fringes we are projecting which are in the horizontal direction. In the following of this paper, we define the object has horizontal movement when the object moves along with $\mathrm{x}$ and has vertical movement when the object moves along with $\mathrm{y}$, and $I_{n}^{o}(x, y)$ denotes the intensity value of n-th fringe pattern image of object, $I_{n}^{r}(x, y)$ denotes the intensity value of $\mathrm{n}$-th fringe pattern image of reference plane, other parameters are distinguished in the same way. The unit for movement distance is pixel and for any display contains $\Delta x$ or $\Delta y$ means the situation that the object already has movement. Assume four-step PSP algorithm is used. The object moves in horizontal direction and vertical direction between the first step and second step will be discussed below. 

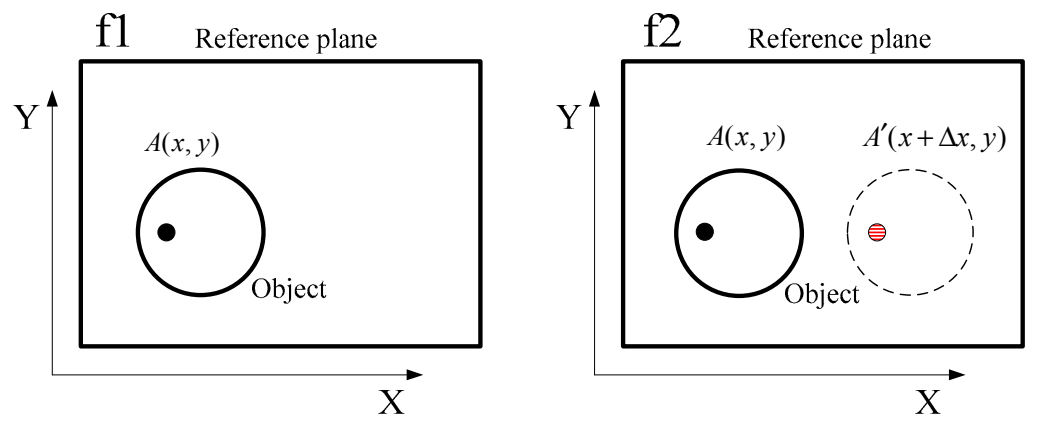

Figure 2. Object moves in horizontal direction

In figure $2, \mathrm{f} 1$ and $\mathrm{f} 2$ is the first and second step respectively, $A(x, y)$ is one point on the object with the coordinate $(x, y)$. If the object moves in horizontal direction which is shown in $\mathrm{f} 2$, the point $A(x, y)$ becomes to $A^{\prime}(x+\Delta x, y)$. Because horizontal fringe patterns are projected, the intensity values on $A(x, y)$ and $A^{\prime}(x+\Delta x, y)$ will satisfy

$$
I_{2}^{o}(x, y)=I_{2}^{o}(x+\Delta x, y)
$$

Once the corresponding relationship of points on object between different steps are determined, $I_{2}^{o}(x+\Delta x, y)$ can be used to calculate the phase value by equation 2 .

2.2.2 Object moves in vertical direction
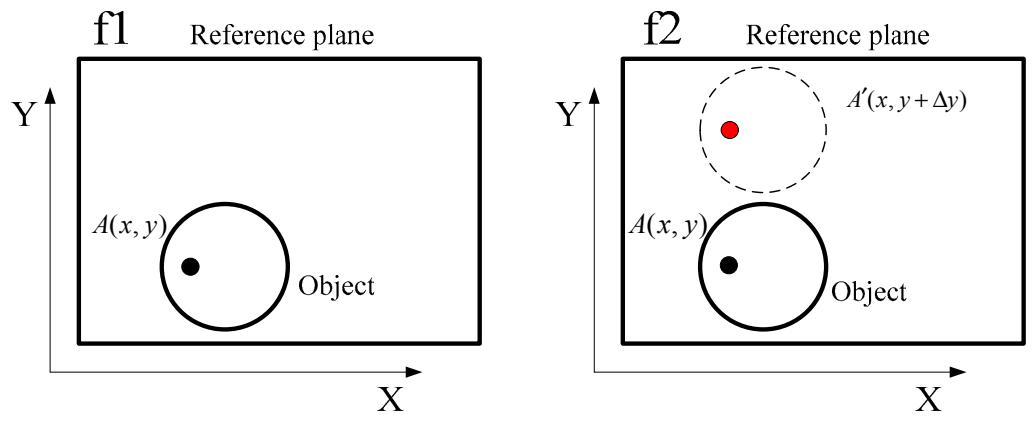

Figure 3. Object moves in vertical direction

Similarly, in figure 3, if the object moves in vertical direction, the point $A(x, y)$ becomes to $A^{\prime}(x, y+\Delta y)$ in $\mathrm{f} 2$. If the movement distance does not equal to integer multiples of the fringe pattern period, the intensity values at $A(x, y)$ and $A^{\prime}(x, y+\Delta y)$ do not equal to each other.

$$
I_{2}^{o}(x, y) \neq I_{2}^{o}(x, y+\Delta y)
$$

Even the corresponding points on the object and movement distance between different steps are known, equation 2 and the intensity value $I_{2}^{o}(x, y+\Delta y)$ cannot be used to calculate the phase value, because the intensity value and phase value of the object are changed by the movement and without phase shift of $2 \pi / N$. In $\mathrm{f} 2$, because the phase shift from $\mathrm{f} 1$ to $\mathrm{f} 2$ is $\pi / 2$, if the object did not move, the phase value in $A(x, y)$ should be $\Phi_{1}^{o}(x, y)+\pi / 2$. Because it is the same point moves from $A(x, y)$ to $A^{\prime}(x, y+\Delta y)$, the height is same, according equation 6 , the unwrapped phase 
difference will satisfy

$$
\begin{aligned}
& \Phi_{2}^{o}(x, y+\Delta y)-\Phi_{2}^{r}(x, y+\Delta y)=\left[\Phi_{1}^{o}(x, y)+\frac{\pi}{2}\right]-\Phi_{2}^{r}(x, y) \\
& \Rightarrow \Phi_{2}^{o}(x, y+\Delta y)=\Phi_{1}^{o}(x, y)+\Phi_{2}^{r}(x, y+\Delta y)-\Phi_{2}^{r}(x, y)+\frac{\pi}{2}
\end{aligned}
$$

Using equation 5 , equation 10 can be expressed as

$$
\phi_{2}^{o}(x, y+\Delta y)=\phi_{1}^{o}(x, y)+\phi_{2}^{r}(x, y+\Delta y)-\phi_{2}^{r}(x, y)+\frac{\pi}{2}+2 \pi k_{\text {sum }}
$$

Where

$$
k_{\text {sum }}=-k_{2}^{o}(x, y+\Delta y)+k_{1}^{o}(x, y)+k_{2}^{r}(x, y+\Delta y)-k_{2}^{r}(x, y)
$$

Equation 11 is the relationship between phase value and movement. The phase value on the object after movement can be expressed by the phase value before movement of the object and the phase values on the reference plane.

Applying equation 11 into all the steps of fringe pattern image, equation 1 for four-step PSP will become to equation 13.

$$
\left\{\begin{array}{l}
I_{1}^{o}(x, y)=I^{\prime}(x, y)+r(x, y) \cos \left[\phi_{1}^{o}(x, y)\right] \\
I_{2}^{o}\left(x, y+\Delta y_{1}\right)=I^{\prime}(x, y)+r(x, y) \cos \left[\phi_{1}^{o}(x, y)+\phi^{r}\left(x, y+\Delta y_{1}\right)-\phi^{r}(x, y)+\frac{\pi}{2}\right] \\
I_{3}^{o}\left(x, y+\Delta y_{2}\right)=I^{\prime}(x, y)+r(x, y) \cos \left[\phi_{1}^{o}(x, y)+\phi^{r}\left(x, y+\Delta y_{2}\right)-\phi^{r}(x, y)+\pi\right] \\
I_{4}^{o}\left(x, y+\Delta y_{3}\right)=I^{\prime}(x, y)+r(x, y) \cos \left[\phi_{1}^{o}(x, y)+\phi^{r}\left(x, y+\Delta y_{3}\right)-\phi^{r}(x, y)+\frac{3 \pi}{2}\right]
\end{array}\right.
$$

For the same point on the object, the average intensity $I^{\prime}(x, y)$ and the intensity modulation $r(x, y)$ are the same among different fringe pattern images. $\Delta y_{1}, \Delta y_{2}$ and $\Delta y_{3}$ are the movement distance for the second, third and fourth step respectively, and are assumed known. $\phi^{r}(x, y)$ can be calculated by fringe pattern images on the reference plane. The unknown parameters are $I^{\prime}(x, y), r(x, y)$ and $\phi_{1}^{o}(x, y)$, we can obtain $\phi_{1}^{o}(x, y)$ by solving equation 13 .

Until now, the relationship between movement and phase value is built. From section 2.2.1 and 2.2.2, it is obvious that only the vertical movement affects the phase value. When the object has two-dimensional movement, the correct wrapped phase value can be calculated by the intensity value after movement under the condition that the corresponding points on object between different steps can be found and the movement distances are known.

\section{SIMULATIONS}

In order to verify the performance of the proposed method, some simulations are finished. We using four-step PSP algorithm simulated a hemisphere. Firstly, the hemisphere is moved in both horizontal direction and vertical direction, and then the object is rotated. We use the proposed compensation method to reconstruct the hemisphere. The simulations are finished under the conditions that the corresponding points on object between different steps can be found, the movement distances are already known. The fringe pattern and reconstruction result of the hemisphere are shown in figure 4. 


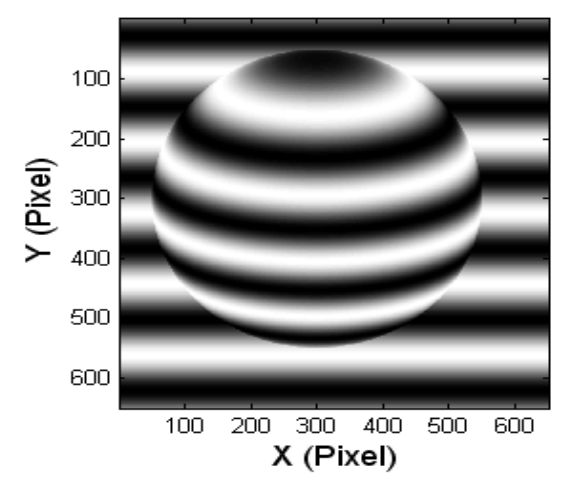

(a)

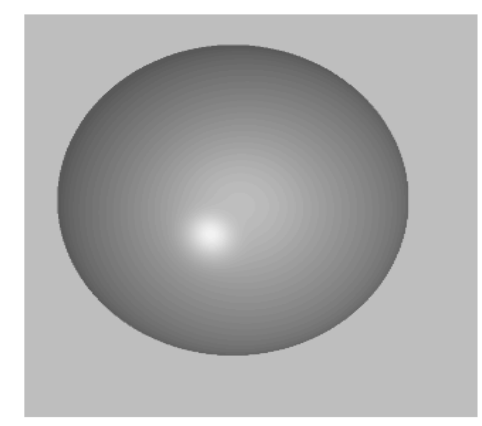

(c)

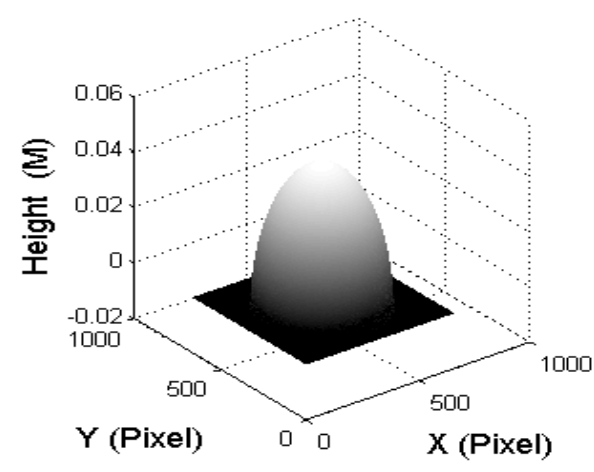

(b)

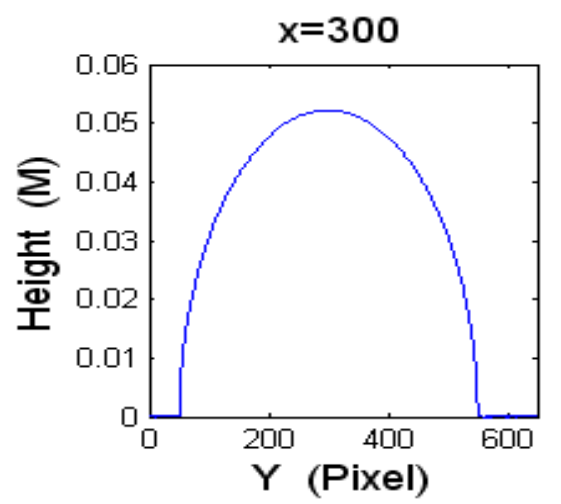

(d)

Figure 4. Hemisphere simulation. (a) Fringe patterns image of hemisphere. (b) Hemisphere reconstruction by Mesh display. (c) Hemisphere reconstruction surface. (d) Cross section for (c) when $\mathrm{x}=300$.

Figure 4(a) is a fringe patterns image of the object, the fringe patterns are distorted by the height of object. Figure 4(b) and (c) are the reconstruction results when the object is motionless. Figure 4(d) is the cross section when $x$ equals to 300 .

When the object moves in both horizontal direction and vertical direction, the reconstruction results without compensation and with compensation are

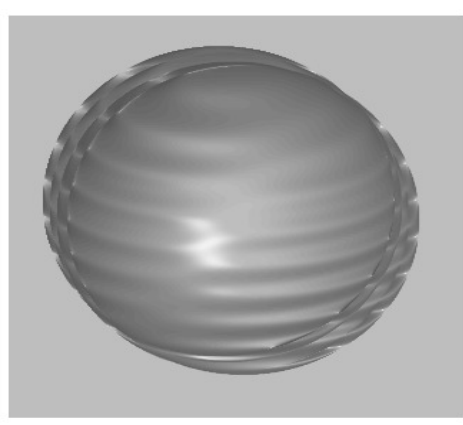

(a)

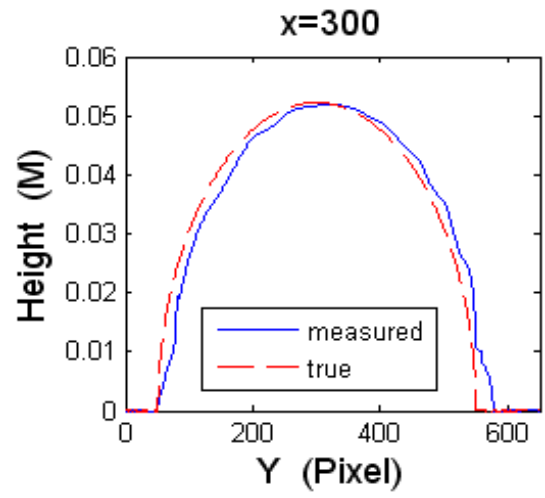

(b) 


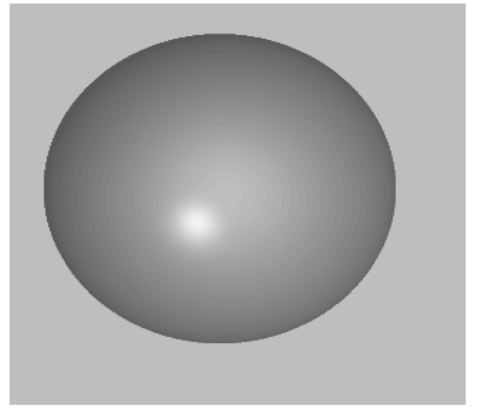

(c)

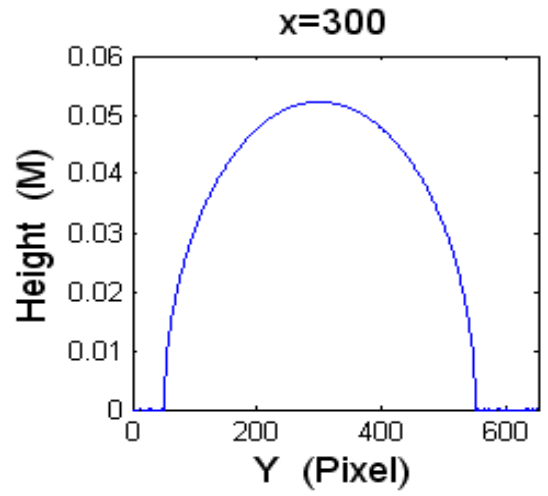

(d)

Figure 5. The object has both horizontal movement and vertical movement. (a) Reconstruction result without compensation. (b) Cross section for (a) when $x=300$. (c) Reconstruction result with compensation. (d) Cross section for (c) when $\mathrm{x}=300$.

The object moves in the bottom right direction from the second step to the fourth step, the total movement distance is 35 pixels in horizontal direction and 30 pixels in vertical direction respectively. There are obvious errors caused by movement without compensation in figure 5(a). In figure 5(b), the red line is the true value when $x$ equals to 300 and the blue line is the result without compensation. After use the compensation method, the object surface can be successfully reconstructed in figure 5(c). The cross sections of true value and measured value are overlapped in figure 5(d).

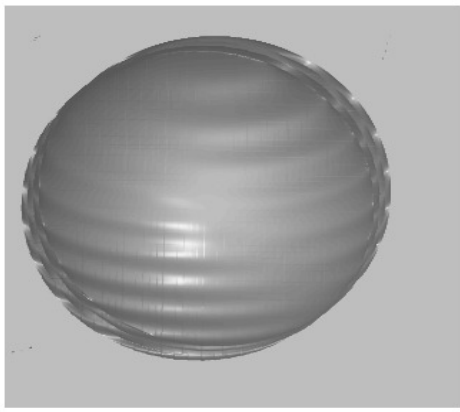

(a)

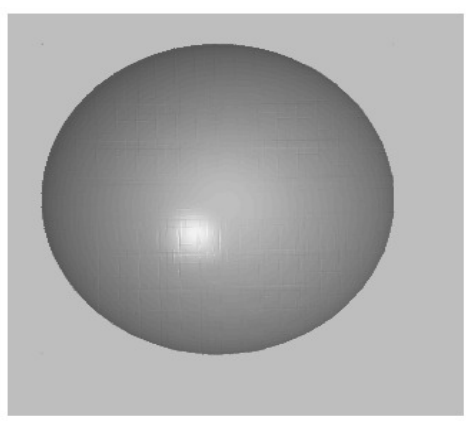

(c)

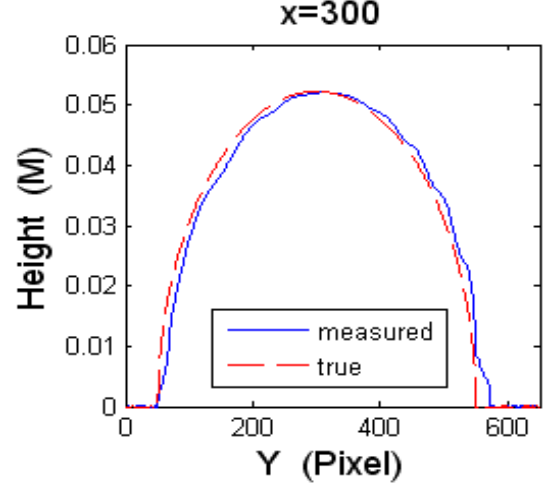

(b)

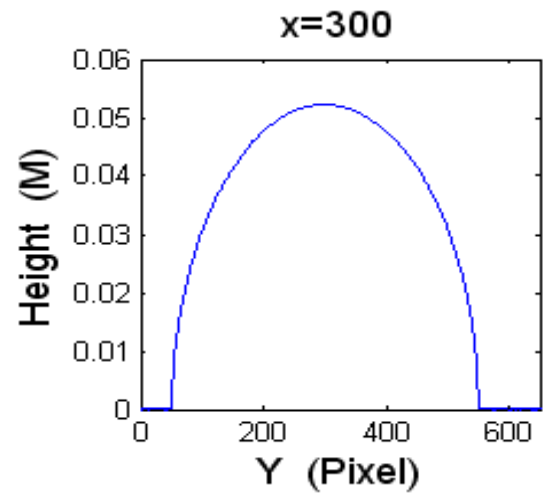

(d)

Figure 6. The object has rotation movement. (a) Reconstruction result without compensation. (b) Cross section for (a) when $\mathrm{x}=300$. (c) Reconstruction result with compensation. (d) Cross section for (c) when $\mathrm{x}=300$. 
It is more common that the object has rotation movement in practice. Figure 6 is the simulation of object has rotation movement. For the specific point on object, the effect of rotation also can be considered as horizontal movement and vertical movement.

The object is rotated counter-clockwise around the up left point in figure 6 (a) from the second step to the fourth step, the total rotation angle is 3 degrees. The range of movement distance in the horizontal direction is from 0 to 10 pixels and from 0 to 41 pixels in vertical direction. Obviously, the reconstruction result with compensation is better than the one without compensation.

\section{CONCLUSION}

In this paper, a compensation method for errors caused by two-dimensional movement of object in PSP is proposed. The compensation method is based on the analysis of the relationship between object movement and phase value. Through the compensation method, the errors caused by two-dimensional movement can be eliminated under the condition that

the movement distances for each point on the object are known, the object with two-dimensional movement can be successfully reconstructed by PSP.

\section{REFERENCES}

[1] M. Takeda, et al., "Fourier-transform method of fringe-pattern analysis for computer-based topography and interferometry," Journal of the Optical Society of America. Paper 72(1), 156-160(1982).

[2] H. C. L. V. Srinivasan, and M. Halioua "Automated phase-measuring profilometry of 3-D diffuse objects," Applied Optics. Paper 23(18), 33105-3108(1984).

[3] L. S. X. Su, W. Li, and L. Xiang, "New 3D profilometry based on modulation measurement," Proceedings of SPIE 3853, 1-7(1998).

[4] X. Su, et al., "Dynamic 3-D shape measurement method based on FTP," Optics and Lasers in Engineering. Papers 36(1), 49-64(2001).

[5] S. Zhang and S. T. Yau, "High-speed three-dimensional shape measurement system using a modified two-plusone phase-shifting algorithm," Optical Engineering. Papers 46(11), 1-6(2007).

[6] Z. Song, "Recent progresses on real-time 3-D shape measurement using digital fringe projection techniques," Optics and Lasers in Engineering. Papers 48(2), 149-158(2010).

[7] K. Liu, et al., "Gamma model and its analysis for phase measuring profilometry," Journal of the Optical Society of America A: Optics and Image Science, and Vision. Papers 27(3), 553-562(2010).

[8] Y. Wang, et al., "3D shape measurement technique for multiple rapidly moving objects," Optics Express. Papers 19(9), 8539-8545(2011).

[9] P. S. Huang and S. Zhang, "3-D Optical measurement using phase shifting based methods," Proc. SPIE 6000, 212 (2005).

[10]S. Zhang, "Digital multiple wavelength phase shifting algorithm," Proc. SPIE 7432, 1-11 (2009). 